\title{
JUSTICIA CIVIL EN LA ERA DIGITAL Y ARTIFICIAL: ¿HACIA UNA NUEVA IDENTIDAD?
}

\author{
CIVIL JUSTICE IN THE DIGITAL AND ARTIFICIAL ERA: \\ TOWARDS A NEW IDENTITY?
}

\begin{abstract}
Álvaro Pérez Ragone*
RESUMEN: La justicia civil como marco público y privado de tutela de los derechos y en especial en el ejercicio de la jurisdicción mediante el proceso judicial se ve desafiada en sus cimientos tradicionales. La incorporación en diversos grados de intensidad de la digitalización y la inteligencia artificial (IA) tendría importantes consecuencias. Los desafíos pueden circunscribirse a: (i) la visibilidad y transparencia del proceso judicial al reducir o eliminar la presencia física del tribunal; (ii) la legitimidad de los tribunales de justicia (o de mecanismos privatizados que los subroguen); (iii) los requerimientos del debido y justo proceso en el sistema de justicia estatal como arbitral, en tanto derecho a una justificación razonable y transparente de la toma de decisiones automatizadas.
\end{abstract}

Palabras clave: digitalización, inteligencia artificial, justicia civil, proceso civil.

ABSTRACT: Civil justice as a public and private framework for the protection of rights and especially in the exercise of jurisdiction through the judicial process is challenged in its traditional foundations. The replacement of lawyers and judges by digitalization and more by artificial intelligence (AI) would have serious implications. The challenges can be limited to: (i) the visibility and transparency of the judicial process and eliminating the physical presence of the court; (ii) the legitimacy of the courts of justice (or the privatized mechanisms that subrogate them); (iii) the requirements of due and fair process in the state and arbitration justice system and process, as the right to a reasonable and transparent justification of automated decision making.

Keywords: Digitalization, artificial intelligence, civil justice, civil procedure.

\section{INTRODUCCIÓN}

La justicia civil puede ser entendida en un sentido macro y en uno micro. El primero comprende los mecanismos privados (donde el juego del rol de la autonomía de la voluntad desempeña el rol principal) como públicos de protección y tutela de derechos. El segundo refiere al cauce de ejercicio de la jurisdicción mediante el proceso civil (estatal o arbitral) los principios, estructuras y dinámicas ${ }^{1}$. La justicia civil estatal varía en modelos comparados

* LL.M. y Dr. Iur (U. Köln), Profesor Titular de Derecho Procesal Civil (Universidad de San Pablo-Tucumán/ Argentina); Investigador Externo Universidad Católica del Norte (Chile). Contacto: Av. Estado de Israel 1062, CP. 4000, Tucumán, Argentina E-mail: alvaro.perez01@ucn.cl. ORCID: https://orcid.org/0000-0002-6069-4564.

${ }^{1}$ Jolowicz (2000) p. 84. 
de sobrecargada a alivianada o con "fuga" de casos a modelos privados independiente del monto de la disputa ${ }^{2}$.

Los sistemas operados por computadoras se utilizan cada vez más para la toma de decisiones en la administración pública, justicia y la empresa privada. Comprenden una variedad de temas técnicos, políticos y controvertidos sobre el acceso y funcionamiento de la justicia. Esta entendida como mecanismos de tutela de derechos, uno de los cuales donde interviene la jurisdicción es el proceso judicial ${ }^{3}$. Las actividades que estaban reservadas a los humanos porque requerían la toma de decisiones en circunstancias variadas e impredecibles ahora pueden ser realizadas por inteligencia artificial (IA). El aprendizaje automático se está desarrollando a un ritmo tal que es concebible que los sistemas operados por algoritmos puedan proporcionar servicios de los procesos judiciales incluso en las resoluciones (decisión judicial) ${ }^{4}$. La sustitución de abogados y jueces por IA tendría serias implicaciones más allá de la pérdida de puestos de trabajo ${ }^{5}$. La justicia civil está bajo presión en grandes y pequeñas causas ${ }^{6}$.

La creciente digitalización de nuestro entorno de vida tiene enormes efectos sobre el Derecho y la aplicación/tutela de los derechos. El impacto real de la digitalización aún está por llegar. Tarde o temprano, tendremos que enfrentarnos a cuestiones fundamentales sobre el diseño del futuro ordenamiento jurídico: ¿Cuáles son las reglas fácticas que rigen las cuestiones del comercio electrónico moderno? ¿Cómo afecta esto a la aplicación e implementación del estado de derecho? ¿Cuáles son las herramientas más adecuadas para satisfacer las demandas legales de los consumidores? ¿Cómo nos sorprende que algún día los abogados-robots comiencen a competir con los abogados y por qué no jueces-robots con los jueces tradicionales? ¿Cómo explicar y hacer comprensibles y legítimas las decisiones (no solo judiciales sino de E-Gobernance)? ¿Hay un derecho a una explicación razonable? Este artículo ofrece una descripción general de las consecuencias de la digitalización y uso de la inteligencia artificial en la justicia, más precisamente en el proceso civil y esboza los desafíos regulatorios más importantes en prospectiva ${ }^{7}$.

Es importante el concepto de transparencia por diseño que sirve como guía práctica para ayudar a promover las funciones beneficiosas en entornos de toma de decisiones automatizada (digitales e inteligencia artificial). Con el auge de la inteligencia artificial (IA) y la capacidad de los sistemas para tomar decisiones automatizadas y auto-aprendidas, se ha hecho eco en los círculos académicos y políticos un llamado a la transparencia sobre cómo estos sistemas toman decisiones ${ }^{8}$. El término transparencia, sin embargo, se relaciona con múltiples conceptos, cumple muchas funciones y encierra diferentes promesas que luchan por realizarse en aplicaciones concretas. De hecho, la complejidad de la transparencia en

\footnotetext{
2 Statistisches Bundesamt (2019) pp. 12 y ss.; Prütting (2018) p. 62; Rühl (2020) pp. 809-817; Calliess (2014) p. 34 y ss.

3 ZuCKerman (2020) pp. 291-300.

4 Legal Tech (2019).

5 Legal Tech (2019).

6 STEFfen (2018) pp. 334 y ss.

7 FRIES (2016).

8 Albu y Flyverbom (2019) pp. 68-297; Buhmann y otros (2019).
} 
este ámbito evidencia una tensión entre un ideal normativo y su traducción en la aplicación práctica ${ }^{9}$. Para abordar esta tensión, se debe hacer una revisión de la transparencia, analizando sus desafíos y limitaciones en relación con las prácticas de toma de decisiones automatizadas, integrándolo como un valor ex ante y no como una ocurrencia tardía. En este tópico nos encontramos en un paso más allá que la sola digitalización de la justicia ${ }^{10}$.

La primera parte de este aporte analiza la situación actual y muestra hasta qué punto ha avanzado la digitalización en la justicia civil (infra II). La segunda examina la inteligencia artificial (IA) (Infra III). Luego se presentan los desafíos de la justicia predictiva o mejor dicho "predecible" (Infra IV). Finalmente se cierra con las conclusiones (infra V.).

\section{DIGITALIZACIÓN DEL PROCESO CIVIL}

La digitalización de la justicia civil ha estado en la agenda de los legisladores y del propio poder judicial durante muchos años. De hecho, durante las últimas dos décadas, se han ido complementando gradualmente los códigos y leyes procesales con disposiciones destinadas a preparar a los tribunales civiles para la era digital ${ }^{11}$. Hasta ahora, la atención se ha centrado en la comunicación y tramitación electrónica. Como ejemplo, la digitalización en Alemania fue paulatinamente establecida por una serie de leyes bajo el título de transacciones jurídicas electrónicas ${ }^{12}$. Así, ya desde el 1 de enero de 2018, ha sido posible, al menos en teoría, comunicarse electrónicamente con todos los tribunales civiles en Alemania ${ }^{13}$. En particular, las demandas y las presentaciones pueden ser electrónicamente presentadas y notificadas por el tribunal (\$\$130a, 169 (4) ZPO). También existe la opción de hacer seguimiento hasta su recepción con confirmación digital (\$174 (3) ZPO).

La conducción y gestión digitalizadas (el e-case management) de los procesos judiciales también se ha convertido en el foco de atención. Los primeros pasos se dieron mediante la apertura de procesos judiciales civiles para transmisiones de audio y video. Desde entonces, las audiencias orales se pueden realizar por videoconferencia previa solicitud -y también por orden del tribunal- de tal forma que las partes, sus representantes autorizados o sus asesores se encuentren en un lugar diferente y la audiencia sea sincrónica ${ }^{14}$. Solo para tomar un ejemplo, en el proceso civil alemán la imagen y el sonido se transmiten a estén los todos participantes y el despacho judicial $(\$ 128 \mathrm{a}(1) \mathrm{ZPO})^{15}$. Además, los testigos y peritos pueden ser escuchados por videoconferencia (\$128a (2) ZPO) y los documentos electrónicos,

\footnotetext{
9 Pégny y otros (2019) pp. 161-178.

10 Campolo y Crawford (2020) pp. 1-19; Edwards y Veale (2018) pp. 46-54; Malgieri (2019).

11 Huber y Giesecke (2020) pp. 2 y ss.

12 Gesetz zu Verwendung elektronischer Kommunikationsformen in der Justiz (2005) p. 837; Gesetz zur Förderung des elektronischen Rechtsverkehrs mit den Gerichten (2013) p. 3786; Gesetz Zur EINFÜHRUNG DER ELEKTRONISCHEN (2017) p. 2208.

13 Gesetz zur Reform des Zivilprozesses (2001) p. 1887; Gesetz Zur AnPassung der Formvorschriften Des Privatrechts und anderer Vorschriften an den modernen Rechtsgeschäftsverkehr (2001) p. 1542; GeSetz zur Reform des Verfahrens bei Zustellungen im gerichtlichen Verfahren (2001) p. 1206.

14 ZuCKerman (2020) pp. 291-304.

15 Gesetz zur Einführung der elektronischen AkTe in der Justiz und ZUr Weiteren Förderung Des elektRONISCHEN RECHTSVERKEHRS (2017) p. 2208.
} 
en particular grabaciones de audio y video, pueden presentarse como prueba (\$371, párrafo 1, oración 2, \$371a, \$371b ZPO). Después de todo, los archivos del caso incluso se pueden conservar de forma completamente electrónica $(\$ 298 \mathrm{a}(1) \mathrm{ZPO})^{16}$. Desde el 1 de enero de 2026, esto deberá -y no simplemente podrá- hacerse (\$298a (1a) ZPO $)^{17}$.

Solo como ejemplos extremos en Latinoamérica vale mencionar a Chile y México. En Chile la regulación del proceso digital se realiza primero por autos acordados o reglamentos de la Corte Suprema y recién luego por ley ${ }^{18}$. En México en el 2017 se dio una reforma constitucional en la cual el Congreso de la Unión tuvo la oportunidad de expedir un código único en materia procesal civil y familiar. Sin embargo, hay obstáculos legales que han ralentizado la transformación digital de la justicia mexicana, lo que ha dificultado el trabajo remoto para los procesos judiciales durante la pandemia por la COVID-19. A pesar de ello, el Tribunal Superior de Justicia del Estado de México no se ha quedado atrás en el desarrollo de mecanismos para la justicia digital en los últimos años ${ }^{19}$. Con la pandemia, la regulación de la justicia digital se ahondó y afianzó, por regla, primero la autoiniciativa del Poder Judicial y luego recién del Legislador ${ }^{20}$.

Por tanto, no se cesado en intentar regular (reglamentaria y/o legislativamente) en los últimos años aspectos de la comunicación judicial y la gestión procesal, teniendo en cuenta el avance de la digitalización en muchos ámbitos. Además, los esfuerzos de digitalización del legislador no se han dirigido hasta ahora -al menos no principalmente- a los ciudadanos que buscan justicia ${ }^{21}$. En tiempos en que las personas hacen casi todo a través de Internet sin pandemias globales, apenas el sistema de justicia civil está actualizado ${ }^{22}$.

Y finalmente el legislador restringe sus esfuerzos de digitalización y no aprovecha plenamente el potencial real que resulta de las posibilidades en constante crecimiento de la tecnología de la información y la informática, en particular de las capacidades de almacenamiento e informática en constante crecimiento, así como de la mejor disponibilidad de datos (Big Data y complementos) ${ }^{23}$.

\section{DesAFíos}

De lo anterior se desprende que muchos sistemas de justicia civil no han llegado realmente al siglo XXI. Si quiere ofrecerse a una sociedad digitalizada una oferta atractiva para resolver disputas a largo plazo, debe, por lo tanto, aprovechar el potencial de la digitalización con mucha más seriedad, independientemente de las pandemias globales ${ }^{24}$. El proceso telemático (que llamaremos "digitalizado") ofrece en su diseño e implementación la

\footnotetext{
16 Greger (2019) p. 3429 y ss.

17 RÜHL (2020) pp. 809-817.

18 Por un lado, la LEY No 20.886 de 2015 y por otro lado, en 2019, se presenta el Instructivo de Transformación Digital para los órganos de la Administración del Estado.

19 Desde la firma electrónica y el Complejo de Transferencia Integrado.

20 Krans, Nylund (2021), pp. 1-5.

21 RÜHL (2020) pp. 809-817.

22 Ver en fallo del Supremo Tribunal Federal alemán en BGH NJW-RR (2009) pp. 357, 358.

23 RüHl (2020) pp. 809-817.

24 Fries (2018); Susskind (2019) p. 15; Ashley (2017) pp. 5-25.
} 
facilidad de que en definitiva se trata de gestionar flujos de procedimientos para los cuales es más que idóneo un proceso judicial con ingreso, tramitación, seguimiento, planificación y decisión como ya lo había expuesto Caponi ${ }^{25}$. Lo que eso significa queda claro cuando se mira fuera de los tribunales. En los últimos años, han surgido aquí ofertas digitales para la solución de controversias y la tutela de derechos, que explotan las diversas posibilidades de la digitalización mucho mejor que el sistema de justicia civil tradicional hasta ahora ${ }^{26}$.

\section{Resolución de Disputas EN LÍNEA}

Las primeras que se mencionan aquí son las ofertas que pueden asignarse al campo de la resolución de disputas en línea (On Line Dispute Resolution, ODR para abreviar). Dependiendo de la intensidad con la que se utilice la tecnología moderna, se pueden distinguir dos generaciones de procedimientos: ODR electrónicos y automatizados ${ }^{27}$.

\section{a) Primera generación: procedimientos $O D R$ electrónicos}

Los procedimientos electrónicos simples de ODR utilizan tecnología para mejorar la comunicación con los involucrados y acelerar el proceso. Las demandas se pueden enviar por correo electrónico o en línea y todo el proceso se realiza por correo electrónico o en línea. Ejemplos de órganos de solución de controversias que aprovechan las ventajas de la digitalización como el caso del arbitraje alemán remoto ${ }^{28}$, el ombudsman austríaco con actuaciones en Internet ${ }^{29} \mathrm{y}$, sobre todo, el órgano general de arbitraje del consumidor de los estados federales alemanes (desde el 1 de enero de 2020: órgano de arbitraje universal) ${ }^{30}$, que es responsable de todo tipo de disputas de consumidores en la implementación de la Directiva Europea de Solución de Controversias ${ }^{31}$. Se puede acceder a él a través de un portal central de Internet, mediante el cual el consumidor puede presentar su queja con la ayuda de una plataforma de entrada bien estructurada en pocos minutos, y a través del cual también se maneja el resto del procedimiento y todas las demás comunicaciones. Desde su inauguración en 2016, la Junta de Arbitraje del Consumidor General alemana, por ejemplo, ha recibido de manera constante un poco más de 2000 solicitudes de arbitraje por año ${ }^{32}$.

\section{b) Segunda generación: procedimientos $O D R$ automatizados}

Los procesos ODR automatizados van un paso más allá. Usan la tecnología no solo para comunicarse más fácil y rápidamente con los involucrados, sino también para resolver disputas como tales ${ }^{33}$. Utilizan técnicas de negociación asistidas por computadora o crean

25 CAPONi (2015) pp. 305-313.

26 SPECHT (2019) p. 153.

27 Braegelmann (2018) p. 215 y ss.

28 Ver sitio web https://www.online-schlichter.de. Fecha de consulta: 10 de febrero de 2021.

29 Ver sitio web https://www.ombudsmann.at. Fecha de consulta: 10 de febrero de 2021.

${ }^{30}$ Ver sitio web https://verbraucher-schlichter.de/. Fecha de consulta: 10 de febrero de 2021.

31 Dir. 2013/11/EU de 21. 5. 2013 Con modificaciones Reglamento (EU) Nr. 2006/2004 y la Directiva 2009/22/EU, ABl. EU 2013, L 165/65.

32 Ver sitio web https://verbraucher-schlichter.de/. Fecha de consulta: 10 de febrero de 2021

33 Carneiro y otros (2014) pp. 211, 215 y ss.; Ashley (2017) pp. 5-25. 
propuestas de comparación que las partes aceptan. El sistema reciente de SmartsettleONE ${ }^{34}$ de la empresa canadiense iCan es considerado disruptivo. En febrero de 2019, en el curso de los procedimientos ante el Tribunal de demandas dinerarias civiles de Inglaterra, este último logró en una hora una solución amistosa de una disputa sobre el pago de una factura de 2.000 libras esterlinas después de que los métodos convencionales de solución de controversias habían fallado previamente.

Los procesos ODR no son las únicas ofertas que entienden cómo utilizar el potencial de la digitalización por sí mismos. A esto se suman las ofertas de los últimos años bajo el eslogan "Tecnología jurídica”, o "Tecnología legal”, Legaltech para abreviar". Utilizan tecnologías digitales para automatizar y simplificar el proceso de búsqueda y aplicación de la ley, y así sucesivamente la esperanza de mejorar ${ }^{36}$. De la plétora de proveedores que han surgido aquí en los últimos años, merecen una mención aquellos que se han dado a conocer bajo el título de asesoramiento jurídico automatizado ${ }^{37}$.

Las actividades se resuelven completamente en línea en muy poco tiempo, lo que demuestra de manera impresionante que la resolución de disputas basada en Internet tiene una buena demanda y al menos funciona a satisfacción de aquellos involucrados en disputas de menor cuantía sin intervención de terceros y autocompositivamente ${ }^{38}$.

$\mathrm{Al}$ mismo tiempo en paralelo, sin embargo, el ejemplo también muestra naturalmente los peligros que surgen cuando los tribunales estatales no logran hacer una oferta atractiva para los pequeños y medianos litigios. EBay y PayPal no están sujetos a ningún control público en lo que respecta a la resolución de disputas, al igual que todos los órganos de resolución de disputas extrajudiciales. En palabras de Hazel Genn: "El resultado [...] se trata solo de un arreglo" 39 .

\section{INTELIGENCIA ARTIFICIAL " EN Y DE LA JUSTICIA CIVIL"}

¿Qué se sigue de todo esto para la justicia civil? ¿Debería reaccionar a las nuevas ofertas de justicia digital? ¿Debería aceptar los desafíos de la digitalización y más profundo de la IA? No cabe duda de que la respuesta es sí. Se podría objetar que el sistema de justicia civil -y el Estado- podrían estar complacidos de que existan proveedores en el campo de las pequeñas y medianas disputas que ayuden a los ciudadanos a buscar justicia y así cerrar la brecha de protección legal que la justicia civil actualmente deja atrás. Esto, sin embargo, malinterpretaría la importancia primordial de hacer valer los derechos con la ayuda de los

\footnotetext{
${ }^{34}$ Ver sitio web https://smartsettle.com/products/smartsettle-one/ Fecha de consulta: 10 de febrero de 2021. Véase también LODDER y THIESSEN (2013).

35 WAGNER (2018) pp. 2-28.

36 WAGNER (2018) pp. 2-28.

37 Wagner (2018) pp. 2-28. Otro ejemplo, casi legendario, de resolución de disputas extrajudicial automatizada es el mecanismo de resolución de conflictos de las empresas de Internet eBay y PayPal, la llamada protección del comprador de PayPal Con su ayuda, supuestamente se desencadenan 60 millones de disputas cada año (Ver al respecto Rule (2017) pp. 354 y ss.)

38 SUSSKIND (2019) p. 98.

39 Genn (2012) p. 16.
} 
tribunales civiles ${ }^{40}$. En contraste con la mayoría de las opciones de implementación extrajudicial, los juicios y litigios civiles de acceso público aumentan nuestro conocimiento de la aplicación e interpretación igualitaria de la ley. Crean seguridad jurídica a largo plazo y confianza en el estado de derecho ${ }^{41}$.

Las preocupaciones sobre los algoritmos de aprendizaje automático de "caja negra" han influido en las leyes y regulaciones modernas de protección de datos en el establecimiento del derecho a la intervención humana en la toma de decisiones con el apoyo de la inteligencia artificial. Tales intervenciones brindan a los interesados los medios para proteger sus derechos, libertades e intereses legítimos, ya sea como un requisito mínimo para el procesamiento de datos o como una norma central que rige la inteligencia artificial para la toma de decisiones ${ }^{42}$. Es importante tener en cuenta acá dos tipos de cuestiones con las implementaciones legales actuales del derecho a la intervención humana: (i) Primero es la incertidumbre sobre qué tipo de decisión debería estar cubierta por este derecho: ¿Debería restringirse la intervención a aquellas decisiones sin participación humana, o bien interpretarse en un sentido más amplio, abarcando todas las decisiones que son efectivamente moldeadas por procesamiento automatizado? y; (ii) Segundo, se deriva de las limitaciones prácticas de este derecho a la intervención: incluso dentro de un marco conceptual claro, los interesados aún pueden carecer de la información que necesitan para el ejercicio concreto de su derecho, o la intervención humana en sí misma puede introducir sesgos y limitaciones que dan lugar a resultados indeseables. Así la protección adecuada de los derechos de los interesados es factible solo si existen medios para controlar y objetar las decisiones basadas únicamente en el procesamiento automatizado de un sistema de inteligencia artificial ${ }^{43}$.

En este sentido, la tecnología ya está cambiando la práctica del derecho y puede, por ejemplo, remodelar el proceso de juzgar reemplazando, apoyando o complementando la función judicial. Dichos cambios pueden limitar la medida en que los seres humanos se dedican a juzgar con un énfasis cada vez mayor en la inteligencia artificial para tratar disputas civiles más pequeñas y el uso más rutinario de tecnologías relacionadas en disputas más complejas $^{44}$. En este sentido, ha habido un creciente enfoque en los tribunales en línea y lo que pueden proporcionar. En la actualidad, pocas de estas propuestas se relacionan con el juez con asistencia de IA y normalmente se centran en aumentar la actividad en línea. Sin un foro público y abierto para el servicio de justicia pro el proceso civil ¿sería aceptado por la ciudadanía el ejercicio de estos poderes? ${ }^{45}$.

Los problemas de base se dan en tres ámbitos: (i) la traducción del derecho en códigos informáticos; (ii) el problema de las decisiones o juicios discrecionales o con problemas de ponderación de valores (hard cases); (iii) el lenguaje judicial (sintaxis y semántica).

40 LOOKS y LOUI (2005).

41 Susskind (2019) pp. 8-10, 140-150.

42 Felzmann y otros (2020) pp. 3333-3361.

43 Felzmann y otros (2020) pp. 3333-3361.

44 Sourdin (2018) p. 1114 y ss.

45 Perry (2017) pp. 29 y ss. 
En relación a lo primero, se ha planteado la cuestión de cómo traducir con precisión la ley en códigos, comandos y funciones que un programa de computadora pueda comprender. El lenguaje jurídico tiene matices y, a menudo, requiere comprensión contextual. Los programadores de computadoras y los profesionales de TI rara vez tienen calificaciones o experiencia legales, ni son expertos en políticas o administrativos. Sin embargo, son estos profesionales quienes tienen la tarea de traducir la legislación y la jurisprudencia en códigos y comandos informáticos para permitir un proceso autónomo para tomar decisiones.

Con respecto a lo segundo, las fuentes del derecho, aunque complejas por sí mismas, también operan en el contexto de presunciones legales y juicios discrecionales. Asegurar que estas complejidades estén codificadas correctamente en un proceso autónomo es un desafío. Debido a estos desafíos, se enfatiza que más áreas regulatorias de la ley pueden ser más adecuadas para ser transformadas en código informático. Muchos juicios dentro del sistema legal involucran un elemento de discreción. Los programas de computadora operan en base a la lógica y tal rigidez es posiblemente incompatible con decisiones discrecionales ${ }^{46}$.

Finalmente, de manera similar, el uso de la IA en el derecho puede enfrentarse a la distinción filosófica entre sintaxis y semántica. Los programas de computadora poseen sintaxis (una estructura formal de operación), pero no poseen semántica (significado detrás de estas operaciones). La tecnología digital procesa información en forma de símbolos abstractos, a saber, unos y $\operatorname{ceros}^{47}$. La tecnología posee la capacidad de procesar y manipular estos símbolos, pero no comprende el significado detrás de estos procesos. En otras palabras, la máquina no comprende la información que está procesando. Esto se puede contrastar con la mente humana, que puede comprender la información que procesa ${ }^{48}$.

\section{AcCeso digital a los tribunales, e-ACCESO a la JUSTiCia}

En la parte superior de la agenda de reformas debería estar la creación de acceso digital actualizado a los tribunales. En particular, se indicaría el establecimiento de un portal de acceso digital y la introducción de procedimientos judiciales (totalmente) digitales. Con ambos, el umbral para reclamar protección judicial se reduciría y la implementación de procedimientos judiciales sería una realidad concreta, no solo en tiempos de pandemias globales.

Por un lado, el material de litigio, como en las páginas de ODR: utilizan máscaras de entrada y cuestionarios (sistemas de orientación legal) bien estructurados para recopilar datos de manera específica ${ }^{49}$. Y por otro, el proceso judicial, al igual que los procedimientos de los proveedores de ODR relevantes, podría llevarse a cabo electrónicamente y, en principio, en una audiencia oral en presencia de todos. Además, el portal de acceso digital y los procedimientos judiciales digitales también podrían mejorarse con procesos automatizados de resolución de disputas, por ejemplo, la opción de subastas judiciales o remates on-line.

Independientemente de estas consideraciones, el establecimiento de un portal de acceso digital y la introducción de procesos judiciales digitales también estarían totalmen-

\footnotetext{
46 Sourdin (2015) pp. 95-116.

47 Galdia (2020) pp. 57-76.

48 SOURdin (2015) pp. 95-100.

49 Felzmann y otros (2020) pp. 3333-3361; Comp. Susskind (2017) pp. 118 y ss.
} 
te en línea con la tendencia internacional. Ello se evidencia porque numerosos países han digitalizado el acceso y el procedimiento ante sus tribunales en los últimos años. En el Reino Unido, por ejemplo, las reclamaciones monetarias de hasta 10.000,00 GBP se pueden hacer cumplir digitalmente a través del portal "Make a money Claim online" 50 . También existe acceso digital a los procesos judiciales digitales en Irlanda. El servicio judicial en línea del poder judicial irlandés se puede emplear aquí para disputas hasta por un valor de $€ 2.000,00^{51}$. Y en Canadá hay incluso un tribunal en línea independiente para demandas de hasta CAD 5.000,00, que lleva a cabo sus procedimientos completamente en línea ${ }^{52}$. En última instancia, el desarrollo está aún más avanzado en Dinamarca. Desde hace algún tiempo, los tribunales civiles solo emplean un portal de acceso digital. Además, los procedimientos judiciales generalmente se llevan a cabo en formato digital ${ }^{53}$. Un trabajo similar está en plena marcha en los Países Bajos ${ }^{54}$.

Sin embargo, la creación de acceso digital a los tribunales y procedimientos judiciales digitales también plantearía problemas. A continuación, se analizan brevemente dos de ellos:

\section{a) Derecho de acceso a la justicia}

El primer problema se relaciona con el portal de acceso digital y se refiere al derecho a la justicia y tutela efectiva, que está garantizado por el estado de derecho en el artículo 20 (3) GG alemana, así como en el artículo 6 del CEDH y el artículo 47 de la Carta de los Derechos Fundamentales de la UE, artículos 8 y 25 de la Convención Interamericana de Derechos Humanos. Requiere que el Estado proporcione un procedimiento público para la resolución de disputas civiles que sea realmente $-\mathrm{y}$ no solo teóricamente- accesible para $\operatorname{todos}^{55}$. A la luz del estilo de vida actual, la creación de acceso digital a los tribunales es un derecho fundamental procesal ${ }^{56}$.

Sin embargo, todavía hay personas que no tienen acceso a Internet o que no pueden (o no saben) utilizarlo. De hecho, algunos informes sobre pobreza y riqueza encontraron que la "proporción de personas 'sin conexión' que no usan Internet y que no planean hacerlo" "solo ha disminuido marginalmente durante varios años" y "queda una buena quinta parte de la población excluida" ${ }^{57}$. Este hecho no obstaculizaría la creación de un portal de acceso digital. Sin embargo, habría que tenerlo en cuenta en el diseño. En particular, a diferencia de Dinamarca, por ejemplo, no debería haber obligación de presentar demandas

\footnotetext{
50 Ver https://www.gov.uk/make-money-claim. Fecha de consulta: 10 de febrero de 2021; Practice Directive 51R - Online Civil Money Claims Pilot; igualmente Practice Directive 51S - The County Court Online Pilot.

51 https://www.csol.ie/ccms/welcome.html). Fecha de consulta: 10 de febrero de 2021; Ver además ZUCKERMAN (2020) pp. 291-304.

52 Ver https://civilresolutionbc.ca/. Fecha de consulta: 10 de febrero de 2021

53 Pirotek (2018).

54 Ver http://www.achmearechtsbijstand.nl/inloggen/Paginas/default. Fecha de consulta: 10 de febrero de 2021

55 RÜHL (2020) pp. 814.

56 SAlter (2017) pp. 112.

57 RÜHL (2020) pp. 814-816.
} 
digitalmente. Por tanto, el uso del portal de acceso digital debe ser opcional, al menos por el momento ${ }^{58}$.

\section{b) Principio de oralidad y publicidad}

El segundo problema se relaciona con los procesos judiciales digitales y se refiere a los principios de oralidad y publicidad, como también, con el derecho de acceso a los tribunales estatales ${ }^{59}$. En el caso de procesos judiciales civiles en primera instancia, generalmente requieren que se celebren procedimientos orales accesibles al público. Por el contrario, los procedimientos judiciales digitales al menos en principio, pueden llevarse a cabo sin audiencia oral y, por tanto, sin participación pública.

Sin embargo, los principios de oralidad y publicidad no se aplican de manera absoluta y sin excepción, ni desde el punto de vista del proceso civil ni desde el punto de vista del derecho constitucional e internacional ${ }^{60}$. Por ejemplo, los tribunales civiles de acuerdo con el $\$ 128$ (2) del ZPO alemán, pueden tomar decisiones sin una audiencia oral en aras de la simplificación y la aceleración, si las partes están de acuerdo. Y en el proceso del tribunal de distrito (Amtsgericht), de acuerdo con el \$495a del ZPO alemán, si el valor en disputa es de $€ 600,00$ solo se puede realizar oralmente a pedido de parte y es admisible una restricción de los principios de oralidad y publicidad con el consentimiento de las partes ${ }^{61}$.

En este contexto, las verdaderas dificultades que supondría la introducción de procesos judiciales digitales se encuentran en el ámbito de la tecnología y la seguridad informática. Permítanme recordar las dificultades iniciales del buzón de correo electrónico especial o el ataque de piratas informáticos que paralizó la Corte de Apelaciones de Berlín durante semanas en 2019 y expuso toda la base de datos judicial al riesgo de filtración y manipulación ${ }^{62}$. Sin embargo, los ejemplos mencionados anteriormente de otros países muestran que es posible superar estas dificultades.

\section{Acceso digital e inteligente al Derecho}

Además del acceso a la justicia, las consideraciones de reforma también deben considerar el acceso a la propia ley. En particular, el sistema de justicia civil debe inspirarse en ofertas en el ámbito extrajudicial y hacer un mejor uso de las posibilidades de la informática y la tecnología de la información, en particular las posibilidades de automatización. Por un lado, el uso de algoritmos deductivos y basados en reglas (sistemas expertos), como lo utilizan actualmente muchas empresas de tecnología jurídica, y, de forma prospectiva, el uso de sistemas inductivos y estadísticos de inteligencia $\operatorname{artificial~}^{63}$.

\footnotetext{
58 RÜHL (2020) pp. 814-816.

59 RÜHL (2020) pp. 814-816.

60 RÜHL (2020) pp. 814-816.

61 RÜHL (2020) pp. 814-816.

62 T-Systems, Forensics Report (2020).

63 RÜHL (2020) pp. 617 y ss.
} 


\section{a) Uso de trabajo deductivo y basado en reglas. Algoritmos (sistemas expertos)}

Los algoritmos deductivos y basados en reglas se caracterizan por el hecho de que están programados explícitamente, es decir, traducen pasos conocidos a priori para resolver una tarea en código de programa. Pueden realizar tareas simples y también complejas que anteriormente realizaban humanos, resolver, pero de forma mucho más fiable, rápida y económica. En la justicia civil alemana, por ejemplo, los algoritmos de este tipo hasta ahora solo se han utilizado en determinados procedimientos automatizados ( $\$ \$ 698$ y siguientes ZPO). Aquí, la corrección formal de las solicitudes de reclamación se comprueba automáticamente ${ }^{64}$.

Sin embargo, con la aparición de las empresas de tecnología jurídica que se describieron, ha surgido la conciencia de que los algoritmos también se pueden utilizar en el poder judicial y también en el área central de la actividad legal, al examinar (y hasta ponderar) cuestiones jurídicas reales. En detalle, se pueden pensar al menos dos posibles usos diferentes: (i) En primer lugar, los algoritmos podrían acoplarse con el portal de acceso digital que se acaba de describir y los procedimientos judiciales digitales de tal manera que las demandas se verifiquen automáticamente para determinar su admisibilidad y su carácter concluyente antes de que se tengan por presentadas ${ }^{65}$; (ii) En segundo lugar, los jueces podrían utilizar algoritmos en el contexto de la toma de decisiones: al examinar la admisibilidad y el fondo de las demandas ${ }^{66}$.

Las oportunidades que traería consigo el uso de algoritmos también se opondrían, por supuesto, a los problemas. Porque, por un lado, incluso responder preguntas jurídicas más pequeñas requiere la programación de sistemas expertos extremadamente complejos. $\mathrm{Y}$, por otro lado, los juristas a veces dudan de que los algoritmos puedan realmente realizar tareas de subsunción de manera satisfactoria. El proceso de subsunción legal es evaluativo, proceso que no es lineal, sino que se desarrolla en "pasos alternos" y con miras al resultado. Por lo tanto, elude una clasificación simple en "dado" o no "dado", como es necesario para el uso de algoritmos. Los defensores contrarrestan esto diciendo que no todos los procesos de suscripción son un proceso de valoración difícil. Y, de hecho, se puede decir: si un vuelo se retrasó o si un apartamento está en un área donde se aplica el límite de precio de alquiler, se puede hacer sin evaluaciones importantes. Por lo tanto, un uso de algoritmos que se limite a los casos correspondientes no debería encontrar ningún problema importante ${ }^{67}$.

Tampoco debería haber preocupaciones sobre el derecho a un juez natural o la independencia judicial en estos casos porque en el uso de la inteligencia artificial (IA) simple los algoritmos solo servirían para apoyar al poder judicial y tampoco serían obligatorios. Así, como depende del juez si confía en los portales de investigación y búsqueda relevantes (impulsados por algoritmos) para encontrar literatura y jurisprudencia, el juez debería tener la libertad de utilizar la ayuda de un algoritmo ${ }^{68}$.

\footnotetext{
64 Bourcier (2011) pp. 37 y ss.

65 Vogl (2018) pp. 53 y 56.

66 Warren (2018).

67 RÜHL (2020) pp. 814-816.

68 Enders (2018) pp. 721 y 723.
} 


\section{b) Uso de trabajo inductivo, predictivo y estadístico.}

La aparición de la inteligencia artificial (IA) está cambiando muchos aspectos de nuestras vidas. El poder judicial no está excluido de este desarrollo. Sin embargo, el uso de la IA hoy y en el futuro previsible se limita a tareas específicas, mientras que el trabajo de un juez requiere una amplia gama de habilidades diferentes. Por tanto, es poco probable que el uso de la IA sustituya por completo el trabajo de los jueces humanos de un día para otro. La introducción de la asistencia de IA al poder judicial probablemente será un proceso lento y gradual que comienza con la existencia paralela de la asistencia de IA y jueces humanos. Se debe tener en cuenta la salvedad del peligro de una renuncia oculta al poder de decisión durante esta fase ${ }^{69}$.

Por otro lado, es más difícil usar sistemas que generalmente se describen como artificialmente inteligentes ${ }^{70}$. Se caracterizan por el hecho de que no están programados explícitamente, sino implícitamente, es decir, no especifican con precisión los pasos individuales que conducen al resultado, sino que reconocen las conexiones y correlaciones entre las variables de entrada y salida de una gran cantidad de datos históricos y luego las traducen en el comportamiento del sistema ${ }^{71}$. Ya se utilizan ampliamente en el sistema del mercado jurídico, especialmente cuando se trata de ver y analizar grandes cantidades de datos. Por ejemplo, los despachos de abogados utilizan el software de análisis para encontrar cláusulas específicas en los contratos que son importantes para la evaluación de riesgos legales. También aprovechan el trabajo de empresas, que se ocupan del análisis estadístico de las sentencias y la predicción basada en datos de las decisiones judiciales ${ }^{72}$.

Por el contrario, el uso de sistemas de inteligencia artificial en el poder judicial ha sido hasta ahora una existencia oscura en la mayoría de los demás países ${ }^{73}$. La única excepción a esto es Estados Unidos. Los tribunales de varios estados federales utilizan sistemas de inteligencia artificial para evaluar la probabilidad de reincidencia de los delincuentes ${ }^{74}$. En el área de la justicia civil, por otro lado, solo, pero al menos, los tribunales de Internet chinos con sede en Beijing y Hangzhou toman medidas "Jueces de IA"75. Estonia también planea tener demandas por un valor de $€ 7.000,00$ decididas por "jueces robots"76. Se conoce el desarrollo de sistemas artificialmente inteligentes que están destinados a apoyar o incluso a reemplazar al juez humano en el área central de su actividad, es decir, en la toma de decisiones. En particular, el desarrollo de sistemas se considera posible y deseable. Encontrar y analizar la jurisprudencia existente para un hecho o cuestión jurídica determinada y redactar sentencias, o al menos redactar un proyecto de sentencia ${ }^{77}$.

69 Buocz (2018) p. 41.

70 Richards y SMART (2016) pp. 5-7.

${ }_{1}$ Millar y Kerr (2016) p. 104; Comp. Rühl (2020) pp. y 617 ss.

72 European Commission for the Efficiency of Justice (2018) pp. 16 y ss.

3 European Commission for the Efficiency of Justice (2018) pp. 16 y ss.

4 ANGWIN y otros (2016).

5 Young (2019).

6 McFadden (2019).

77 Aylwin (2016). 
Sin duda estos sistemas ofrecerían muchas ventajas para el poder judicial, a los propios abogados y la inclusión de los $\mathrm{ADR}^{78}$. Por un lado, podrían ayudar a evitar errores y, por otro, asegurar la unidad del ordenamiento jurídico y el cumplimiento del principio de igualdad. Al mismo tiempo, podrían ayudar a acelerar los procedimientos y aliviar al poder judicial. Sin embargo, lo que suena tentador en teoría se enfrenta a desafíos considerables en la práctica, al menos hasta ahora ${ }^{79}$. Aparte del hecho de que todavía no existen sistemas que puedan siquiera asumir parcialmente la actividad judicial o complementarla de manera significativa. Mientras tanto es bien sabido que la inteligencia artificial puede discriminar a las personas de manera indeseada $\mathrm{y}$, en determinadas circunstancias, legalmente inadmisible ${ }^{80}$. Además, los sistemas relevantes generalmente se presentan como cajas negras, la forma en que funcionan para el usuario, y generalmente para ellos mismos, para el desarrollador no es (completamente) comprensible: el sistema se alimenta de datos y al final arroja un resultado sin que sea posible explicar qué sucede exactamente en el medio ${ }^{81}$.

\section{Desafíos de la IA EN EL PROCESO CIVIL}

No hace falta decir que estos problemas deben resolverse antes de que la inteligencia artificial pueda utilizarse en el poder judicial. Por lo tanto, se deben realizar esfuerzos para desarrollar sistemas que protejan los derechos humanos como fundamentales y eviten discriminaciones inaceptables ${ }^{82}$. Además, se debe garantizar que los sistemas relevantes funcionen con transparencia y que los resultados sean comprensibles para que puedan ser controlados por el juez y los justiciables. Así, se puede justificar un derecho a una inferencia razonable ${ }^{83}$. Los enfoques de investigación, que recientemente han impactado bajo el eslogan IA deben aún profundizarse y expandirse, están dirigidos a desarrollar mecanismos que aumenten artificialmente la transparencia y la comprensibilidad ${ }^{84}$. Los sistemas inteligentes deberían asegurarse, por ejemplo, mediante la creación de los llamados mapas de prominencia que muestren qué datos fueron importantes o menos importantes para el resultado logrado ${ }^{85}$.

Por último, también habría que garantizar que se preserven los derechos procesales fundamentales pertinentes, a saber, el derecho a un juicio justo, así como la independencia judicial ${ }^{86}$. Deben preservarse los resultados y recomendaciones del sistema. En virtud de estas consideraciones: (i) por un lado, el juez no estaría obligado, por lo que tendría que retener la decisión final ${ }^{87}$; (ii) por otro lado, el peligro del sesgo de la automatización

78 DALKE (2013) pp. 703, 704.

79 WACHTER Y otros (2017).

80 BALKIN (2015) pp. 45, 59. Por ejemplo, el sistema COMPAS (Correctional Offender Management Profiling for alternative Sanctions) los hombres negros tienen más del doble de probabilidades de tener un riesgo de recaída por error que los hombres blancos.

81 Ver LARSON y otros (2016).

82 European Commission for the EfFiciency of Justice (2018) nota 92.

83 European Commission for the Efficiency of Justice (2018) pp. 16 y ss.

${ }^{84}$ BRand (2020) pp. 115-132.

85 BusCH (2018).

${ }^{86}$ Burrell (2016) p. 1.

87 European Commission for the Efficiency of Justice (2018) nota 92. 
tendría que ser contrarrestado y debería asegurarse que los jueces no acepten automática y ciegamente los resultados de la computadora en casos individuales sin un examen crítico ${ }^{88}$. El diseño apropiado de los sistemas artificialmente inteligentes, así como la capacitación, la información y la educación, tendrían que poner a los jueces en una posición y ser alentados a tratar críticamente las propuestas de decisiones automatizadas. Además, no debe haber duda de que, en última instancia, es el juez, y no el sistema utilizado el responsable de las decisiones tomadas ${ }^{89}$.

Así, se plantea en este aporte una posición escéptica ante las preocupaciones sobre la opacidad de las herramientas de decisión algorítmica. Si bien la transparencia y la posibilidad de explicar justificadamente son sin duda importantes en la gestión algorítmica, debiera preocupar que la toma de decisiones automatizada se mantenga en un estándar alto, posiblemente debido a una estimación irrealmente alta del grado de transparencia que se puede obtener de los tomadores de decisiones humanos ${ }^{90}$. En realidad, gran parte de la toma de decisiones humana está plagada de problemas de transparencia, mostramos en este trabajo aspectos en los que a la IA le va un poco mejor, y argumentando que al menos algunas propuestas regulatorias podrían terminar poniendo el listón más alto que es necesario o de hecho útil ${ }^{11}$. No se debe esperar que las herramientas de decisión que apoyan o reemplazan el razonamiento práctico apunten a un estándar más alto que este. En la práctica, esto significa que el tipo de explicaciones para decisiones algorítmicas que son análogas a las de posturas intencionales deben preferirse a las que apuntan a las entrañas arquitectónicas de una herramienta de decisión ${ }^{92}$.

Debe ser posible mantener los estándares de procedural fairness. Los criterios de equidad algorítmica para los modelos de aprendizaje automático están generando un interés de investigación generalizado ${ }^{93}$. Ello en especial por tratarse dentro del contexto de datos personales en línea y muchas veces con procesos algorítmicos opacos y la necesidad de establecer los estándares éticos y jurídicos para la legitimidad del sistema y la confianza del usuario final ${ }^{94}$. Las buenas prácticas de respeto al debido y justo proceso que se integra por la transparencia debieran contemplar ${ }^{95}$ : (i) la contemplación de la persona también a partir de valores especiales, minoritarios y no solo de los estereotipos mayoritarios; (ii) usar datos para demostrar la diversidad dentro de una persona y del grupo al que pertenece; (iii) explicar los métodos y sus limitaciones tecnológicas y algorítmicas en forma accesible y transparente; y (iv) triangular la información para aumentar la veracidad y credibilidad de la información ${ }^{96}$. A medida que los algoritmos asumen roles cada vez más gerenciales-decisionales y de gobierno, es cada vez más importante construirlos para que sean percibidos como justos

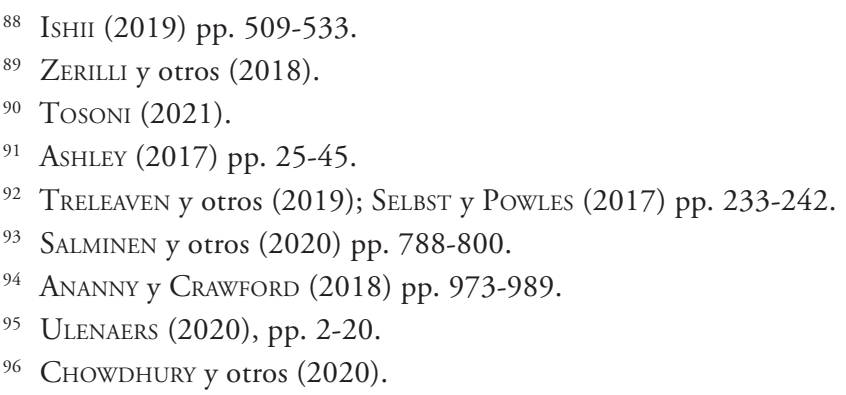


y adoptados por las personas. Con este objetivo, es posible también acá pensar un marco de justicia o equidad procesal en la toma de decisiones algorítmica ${ }^{97}$.

\section{IV. ¿JUSTICIA PREDICTIVA "PREDECIBLE"?: DERECHO A LA TRANSPARENCIA ALGORÍTMICA}

Resulta central la construcción de datos abiertos para las decisiones judiciales y la relación entre esta y la llamada justicia "predictiva", así como las consecuencias de esta última en el ejercicio de la abogacía y la justicia. Se debe hacer una precisión semántica crucial de inmediato. La expresión "justicia predictiva”, es una mala traducción de la fórmula anglosajona predictive justice que se traduciría más correctamente en "justicia predecible" 98 . De hecho, en la actualidad, no se trata, a través del software, de conducir en un instante a una decisión judicial estándar que el juez solo tendría que firmar, ni siquiera a un “juez robot”, sino solo analiza masivamente decisiones judiciales anteriores para identificar tendencias en un caso específico ${ }^{99}$. Desafortunadamente, no puede dejarse al margen cuestionamientos como: ¿No es eso para fines de marketing y publicidad? Esta es la primera fórmula que fue adoptada y ampliamente difundida, esta que ha alimentado muchas fantasías y temores entre los juristas ${ }^{100}$.

En muchos países por el momento los datos abiertos (Open Data) aún no se consideran en la actualidad debido a la falta de difusión de casos y referencias en las decisiones de los tribunales supremos ${ }^{101}$. Como la "justicia predictiva” requiere necesariamente el acceso a tantas decisiones como sea posible para ser eficaz, todavía está lejos de ser muy utilizada.

Austria es uno de los países más avanzado de los términos de datos abiertos ya que cuenta con un portal llamado "RIS" (Rechtsinformationsystem des Bundes) donde cualquier individuo puede acceder, en principio, a cualquier decisión judicial austriaca ${ }^{102}$. Sin embargo, esta base de datos debe ser alimentada por los propios tribunales, y no por el gobierno federal o los estados federales. La consecuencia es que en la práctica los tribunales no tienen ni los medios ni el tiempo para proceder con este envío y, en ausencia de obligación para ellos, no lo hacen, lo que implica que este portal deviene en un espejismo de los datos abiertos.

Francia, por su parte, está a la vanguardia de los países de Europa continental en términos de "justicia predictiva”, ya que existen varias Legaltech que están desarrollando este tipo de herramientas y están comenzando a comercializarlas, y parecen estar en el camino correcto para cuales son los datos abiertos de las decisiones judiciales. Incluso ya una ley del 2016 regula la "República numérica"103 que ha previsto efectivamente esta herramienta para dar a conocer al público todas las decisiones de los tribunales judiciales y administrativos de los tres niveles, aunque las decisiones de los tribunales supremos (Consejo Consti-

\footnotetext{
97 LEE (2019) pp. 1-24.

98 Dondero (2017) p. 532; Cottin (2019) p. 293; Thevenot (2019) pp. 321 y s.

99 Mitrou (2019).

100 Ashley (2017) pp. 20-50.

101 Buat-Ménard y Giambiasi (2017) p. 1483.

102 Ver sitio web de Rechtsinformationsystem des Bundes.

103 Lor No 2016-1321 del 7/10/2016 "République numérique".
} 
tucional, Tribunal de Casación y Consejo de Estado) ya son objeto de una provisión total y gratuita. Sin embargo, las modalidades de realización de esta big data siguen siendo desconocidas ya que aún no se ha publicado el decreto de implementación, de ahí el misterio en cuanto a su forma y su contenido (¿de qué se tratará? ¿Que las decisiones publicadas serán anonimizadas o "seudonimizadas"?) permanece intacta. Cómo juega la publicidad de la información (publicidad del proceso y la justicia) ${ }^{104}$.

Parecería que, en algunos modelos como el austriaco o el alemán, no les preocupa la difusión de los nombres de los magistrados en las decisiones publicadas en línea, ya que, en sus respectivas legislaciones, existe una pantalla bastante considerable para la elaboración de perfiles: principios constitucionales precisan sobre la distribución de casos entre los magistrados. Estos principios establecen un mecanismo que parece bastante idéntico entre los dos Estados y que se basa en una ausencia total de compartimentación de los asuntos. Cualquier juez puede ocuparse de todos los asuntos, lo que ya complica la elaboración de perfiles ${ }^{105}$.

En realidad, la elaboración de perfiles de jueces (de hecho en el foro o sistematizada por publicaciones) ya existía, pero no de forma automatizada como sería el caso de la "justicia predictiva". Lo que se llama trivialmente "ruido de pasillo" es un lugar común en los foros entre abogados y todo profesional sabe que tal o cual juez tiende a juzgar de cierta manera ${ }^{106}$. Pero esto es solo una práctica informal y basado en una experiencia y un sentimiento humanos, mientras que la "justicia predictiva” permitiría saber de una manera determinada y precisa cómo, en un caso determinado, decide el juez X y qué cantidad otorga en promedio. Este conocimiento profundo de la jurisprudencia y del sujeto decisor puede tener varias consecuencias, que discutimos a continuación, en forma de una clasificación de pros y contras (aunque muchas de estas consecuencias se incluyen en ambos extremos) ${ }^{107}$.

\section{1. ¿JUEZ PREDECIBLE?}

Saber exactamente cómo y por qué los magistrados, en un tema dado y en un caso específico, juzgan como juzgan permitiría a los investigadores comprender las tendencias en la jurisprudencia y anticipar mejor los posibles cambios en la posición de los tribunales supremos. Sin embargo, este conocimiento total de la labor de un magistrado puntual a lo largo de su carrera daría a los abogados armas muy poderosas para defender sus reclamos ante dicho magistrado. Podrán "presionar" a este último recordándole que ha estado juzgando en este sentido durante mucho tiempo y que ha concedido tanto, por lo que le resultaría incomprensible juzgar lo contrario en un caso similar que tiene que juzgar ahora ${ }^{108}$.

La cuestión de la valentía de los jueces se puede ver a la inversa: el abogado, pensando que el magistrado cederá a las presiones del pasado, le presenta cifras y casos similares, pero no idénticos; el magistrado podría entonces rebelarse y juzgar de manera completa-

\footnotetext{
104 CADiet (2017).

105 Cottin (2019) p. 293 y ss.; Zwickel (2018), pp. 179-183.

106 Teuber (2018) pp. 155, 198 y ss.

107 Susskind (2019) pp. 100-130; WaLdman (2019) pp. 613-632.

108 Günther (2016) pp. 251 y ss.
} 
mente diferente. Sin embargo, esto sigue siendo poco probable debido a la falta de tiempo de los magistrados y su renuencia a que sus decisiones sean censuradas por tribunales superiores por incumplimiento de la igualdad de trato a y entre los litigantes ${ }^{109}$.

Si todas las decisiones, tan pronto como se dictan, se publican, la revisión por parte de los tribunales supremos de la legalidad de las decisiones inferiores podría cobrar mucha importancia. Esta pregunta en Francia gira por ejemplo en torno a la Corte de Casación que se creó originalmente solo para censurar decisiones dictadas ilegalmente por jueces de apelación. En la actualidad, la Corte de Casación solo conoce de estas sentencias si son objeto de recurso de casación. Por lo tanto, la publicación de todas las decisiones podría permitir a los jueces de derecho supervisar con más detalle todas las decisiones de los tribunales de apelación, incluso las que satisfacen a todas las partes. Sin embargo, esta hipótesis no parece deseable, ya que si la decisión le conviene a todos: ¿Qué interés habría en censurarla y enviar a todos a una audiencia más? Aunque esto es en principio un beneficio porque el respeto a la ley estaría mucho mejor asegurado que antes, es poco probable que los magistrados del tribunal de Casación tengan tiempo para comprobar la legalidad de todos los recursos ${ }^{110}$.

\section{DeRECho a UnA JUSTIFICACIÓN Y EXPLICACIÓn ALGORÍTMICA RAZONABLE}

A medida que aumentan las preocupaciones sobre la injusticia y la discriminación en los sistemas de aprendizaje automático de "caja negra", ha surgido un verdadero "derecho a una explicación” jurídica justificada como un enfoque convincentemente atractivo para impugnar ${ }^{111}$. Si bien los derechos individuales pueden ser útiles, en el derecho de la privacidad históricamente han sido una carga irrazonable en el sujeto promedio ${ }^{112}$. La "información significativa” sobre la lógica algorítmica es técnicamente más posible de lo que se piensa comúnmente, pero esto exacerba una nueva "falacia de transparencia": una ilusión de remedios en lugar de algo sustancialmente útil. Si bien los enfoques basados en derechos merecen un lugar firme en la caja de herramientas, otras formas de gestión y políticas públicas al respecto, como evaluaciones de impacto, "soft law", revisión judicial y repositorios de modelos, merecen más atención, junto con agencias catalizadoras que actúan para que los usuarios controlen fácil y efectivamente el diseño de sistemas algorítmicos ${ }^{113}$.

Muchos han pedido responsabilidad algorítmica: leyes que rijan la toma de decisiones mediante algoritmos complejos o inteligencia artificial. El Reglamento General de Protección de Datos de la UE (GDPR) ahora establece exactamente esto. El reciente debate sobre el "derecho a la explicación" (un derecho a la información sobre las decisiones individuales tomadas por algoritmos) ha oscurecido el importante régimen de responsabilidad algorítmica establecido por el GDPR. Las disposiciones sobre responsabilidad algorítmica,

\footnotetext{
109 Gregor (2019) pp. 267 y ss.

110 SaUvé (2018) p. 7.

111 En general para E-Gobernance ver PÉGNY y otros (2019) pp. 161-178.

112 WaCHTER y otros (2017) pp. 76-99.

113 Edwards y Veale (2018) pp. 46-54
} 
que incluyen el derecho a una explicación, tienen el potencial de ser más amplias, más sólidas y más profundas ${ }^{114}$.

Los datos abiertos de decisiones acoplados a la "justicia predictiva" no solo tendrían inconvenientes, de lo contrario no sería el mercado en crecimiento que es hoy. La primera de estas ventajas es el anverso de la moneda de estandarización de decisiones, que volvería mecánico el trabajo del juez y que se vería favorecido por la falta de coraje y desenvoltura como hemos visto antes ${ }^{115}$. Este otro borde de la estandarización es la potencial reducción de las desigualdades. Dado que la "justicia predictiva” permitiría saber en cada tribunal si, en un caso específico, el actor ha tenido éxito y qué medidas tomó el juez, los abogados podrían hacerlo fácilmente ${ }^{116}$. Promover el argumento de la igualdad entre los ciudadanos para obtener una solución similar a la tendencia general. Esto eventualmente conduciría a un aumento de nivel, ya que todos los abogados se basarían en el rango superior de la tendencia y los jueces encontrarían cada vez más difícil oponerse a esa tendencia, por temor a ser censurados, por provocar una brecha de desigualdad entre los litigantes ${ }^{117}$.

Finalmente, y este es uno de los argumentos de venta más utilizados por legaltech, el conocimiento de las probabilidades de éxito y de los montos otorgados (porque esto es lo que muchas veces interesa principalmente a los litigantes) de forma anticipada serían un factor muy persuasivo en el uso de la resolución alternativa de disputas (ADR) ${ }^{118}$. El abogado podría dar cifras bastante seguras sobre las posibilidades de éxito del caso ante el tribunal de que se trate y las cantidades que el cliente podría esperar, lo que le daría lo suficiente para convencerlo de que es mejor intentar negociar directamente con el oponente en caso de que estos números no estén a su favor. Incluso si las posibilidades son medias, la pregunta puede remitirse a los plazos: ¿Es mejor tener un 55\% de posibilidades de ganar y obtener una compensación completa por todos sus daños pero esperar unos dos años? o ¿ Si no hay apelación, o acepta reducir un poco su reclamo y se resuelve amistosamente en menos de seis meses? ${ }^{119}$ Incentivos negativos y positivos en definitiva para tomar una decisión.

\section{LA TRANSPARENCIA COMO REQUERIMIENTO DEL DEBIDO PROCESO DIGITAL Y ARTIFICIAL}

A medida que la inteligencia artificial y el análisis de big data reemplazan cada vez más la toma de decisiones humanas, las preguntas sobre la ética algorítmica se vuelven más urgentes. A muchos les preocupa que una sociedad algorítmica sea demasiado opaca para ser responsable de su comportamiento. A una persona se le puede negar la libertad condicional o el crédito, despedirla o no contratarla por razones que nunca conocerá y que no se pueden articular. En el sector del proceso judicial, la opacidad de la toma de decisiones al-

\footnotetext{
114 KaMinSKi (2019) pp. 189-217.

115 Garapon (2017) p. 31.

116 EdWARdS y Veale (2018) pp. 46-54.

117 Ferrand y otros (2019) p. 15 y ss.; Comp. en general con Pégny y otros (2019) pp. 161-178.

118 Giacalone (2016); Fersini y otros (2014) pp. 228-236.

119 Comp. Krause (1993) pp. 249-263.
} 
gorítmicas es particularmente problemática, tienen deberes especiales de responsabilidad ${ }^{120}$. Probar los límites de la transparencia en torno al despliegue gubernamental (y en especial de administración de justicia) de análisis de big data, contribuyendo a la literatura sobre responsabilidad algorítmica con un estudio exhaustivo de la opacidad de los algoritmos predictivos gubernamentales. Usando procesos de registros abiertos, enfocamos nuestra investigación en la implementación de algoritmos predictivos. Y es aquí donde las agencias limitadas tienen más probabilidades de entregar el análisis de datos a proveedores privados, que pueden tomar decisiones de diseño y políticas invisibles para las agencias clientes, el público o ambos $^{121}$.

La cuestión de la equidad algorítmica más discutida ha sido si es probable que los algoritmos predictivos introduzcan o perpetúen una discriminación injusta por motivos de raza, género u otra característica protegida. Las formas adicionales de discriminación son motivo de preocupación, como si un algoritmo incidentalmente desfavorece (y por tanto, desincentiva) determinadas conductas. La opacidad algorítmica es un problema ampliamente reconocido y definido de diversas maneras. Algunos de los desafíos comunes mencionados incluyeron garantizar la validación y autenticación necesarias de la información, así como la seguridad de los datos. Al mismo tiempo, un acceso más fácil a la información y, por lo tanto, la transparencia del sistema judicial no solo ha sido importante en sí mismo, sino que también ha aumentado la calidad de la justicia. El acceso para monitorear el avance de los procedimientos, bases de datos legales de buena calidad y la disponibilidad de versiones públicas de las sentencias también ha brindado una oportunidad para la conciencia pública y el escrutinio, mejorando la rendición de cuentas de todo el sistema judicial. También han simplificado la recopilación de información estadística y la medición del progreso y los resultados ${ }^{122}$.

Las explicaciones contra-fácticas son un ejemplo destacado de métodos de interpretación post-hoc en el campo de investigación explicable de la IA. Surgen de otros tipos de explicaciones, ya que brindan a las personas escenarios alternativos y un conjunto de recomendaciones para lograr el resultado deseado del modelo de aprendizaje automático ${ }^{123}$. Recientemente, la literatura especializada ha identificado desiderata de explicaciones contra-fácticas, como viabilidad, accionabilidad y escasez, que deberían apoyar su aplicabilidad en contextos del mundo real. Sin embargo, se ha descuidado el problema de la dependencia del tiempo de las explicaciones contra-fácticas. Así es posible argumentar que, debido a su dependencia del tiempo y debido a la provisión de recomendaciones, incluso las explicaciones contra-fácticas reales, procesables y escasas pueden no ser apropiadas en aplicaciones del mundo real. Esto se debe a la posible aparición de lo que llamamos "eventos contrafácticos desafortunados" ${ }^{124}$.

\footnotetext{
120 Brauneis, Goodman (2018) p. 103 y ss.

121 Ananny y CraWford (2016) pp. 1-20.

122 Citron (2008), pp. 1249 y ss.

123 Ferrario y otros (2019) pp. 523-539; Kaushik y otros (2020).

124 SAMEK y MÜller (2019) pp. 5-22.
} 
Estos eventos pueden ocurrir debido al reentrenamiento de los modelos de aprendizaje de la máquina cuyos resultados deben plantearse a través de explicaciones contrafácticas. Una serie de eventos contra-fácticos desafortunados frustran los esfuerzos de aquellos individuos que implementaron exitosamente las recomendaciones de explicaciones contra-fácticas. Esto afecta negativamente la confianza de las personas en la capacidad de las instituciones para proporcionar decisiones respaldadas por el aprendizaje automático de manera consistente ${ }^{125}$.

\section{CONCLUSIONES}

La Cuarta Revolución Industrial no sigue una trayectoria de desarrollo lineal, pero debido a la naturaleza diversa y al rápido ritmo de los desarrollos tecnológicos, podría compararse más bien a una serie de redes con múltiples puntos de conexión ${ }^{126}$. Esto ha provocado que el desarrollo del derecho (en especial la ley formal), que se ocupa de estas preocupaciones, sea generalmente lento e incapaz de igualar el ritmo y el alcance de los avances tecnológicos ${ }^{127}$. En el contexto del derecho público, hay muchas cuestiones y desafíos relacionados con los derechos individuales, por ejemplo, el derecho a la privacidad y el papel y las responsabilidades del gobierno en relación con el desarrollo de políticas y la regulación que se ocupan de la Cuarta Revolución Industrial ${ }^{128}$.

Podría decirse que el concepto de Rechtsstaat podría proporcionar un marco legal apropiado para dar forma al marco ético, los estándares normativos y un modelo de gobernanza basado en valores para la Cuarta Revolución Industrial, incluso para la toma de decisiones algorítmica. El concepto de rendición de cuentas de derecho debe contextualizarse para aplicarlo a la toma de decisiones algorítmicas ${ }^{129}$. Se concluye que se necesita un amplio conjunto de principios éticos y legales que puedan guiar el desarrollo de marcos legales nacionales e internacionales para regular la toma de decisiones algorítmicas ${ }^{130}$. Muchos de ellos son nuevos componentes del debido y justo proceso.

La digitalización es uno de los grandes desafíos de nuestro tiempo. Tampoco debería detenerse en la justicia civil. Por lo tanto, es de agradecer que el legislador (y el propio poder judicial) haya comenzado hace muchos años a enriquecer con disposiciones sobre transacciones legales electrónicas, por un lado, sobre la conducción electrónica de procedimientos legales, y por fin (aunque mucho menos) sobre la inteligencia artificial aplicada al proceso civil. Sin embargo, los ciudadanos que buscan el derecho apenas reciben ninguna de las muchas opciones. Además, los diversos potenciales de la digitalización aún no se han utilizado seriamente.

A medio y largo plazo también deberían desarrollarse algoritmos y sistemas de inteligencia artificial que puedan ayudar a los tribunales en su toma de decisiones. Por supuesto,

\footnotetext{
125 Doshi-Velez y Kim (2017); Ferrario y Loi (2020).

126 ZARSKY (2013) pp. 1503-1570.

127 SCHWAB (2016).

128 LOOKS y Loui (2005).

129 Fries (2016).

130 Buttarelli (2018).
} 
habrá que discutir y examinar mucho más detalladamente. En particular, será necesario sondear en qué medida los procesos judiciales civiles pueden digitalizarse de manera coherente de conformidad con los requisitos constitucionales y qué requisitos deben cumplir los algoritmos y los sistemas de inteligencia artificial para que puedan ser utilizados en el poder judicial.

\section{BIBLIOGRAFÍA CITADA}

Albu, Oana y Flyverbom, Mikkel (2019): "Organizational transparency: Conceptualizations, conditions, and consequences", Business and Society, vol. 58, No 2: pp. 268-297.

Ananny, Mike y Crawford, Kate (2018): "Seeing without knowing: Limitations of the transparency ideal and its application to algorithmic accountability”, New Media \& Society, No 20: pp. 973-989.

Angwin, Julia y otros (2016): "Machine Bias", ProPublica. Disponible en: https://www. propublica.org/article/machi ne-bias-risk-assessments-in-criminal-sentencing. Fecha de consulta: 20 de febrero de 2021.

Ashley, Kevin (20127): Artificial Intelligence and Legal Analytics: New Tools for Law Practice in the Digital Age (Cambridge, Cambridge U. Press).

Aycwin, Nicole (2016): "Human Centred Design and the Justice System". Disponible en: http://www.slaw.ca/2016/06/06/human-centered-design-and-the-justice-system-lessonsfrom-the-field/. Fecha de consulta: 20 de febrero de 2021.

Balkin, Jack (2015): “The Path of Robotics Law”, California Law Review, No 6: pp. 45-59. Bourcier, Daniéle (2011): "L'acte de juger est-il modélisable? De la logique à la justice”, en L'E-justice. Dialogue et Pouvoir, coll. Arch. phil.dr., t. 54 (Paris, Dalloz) pp. 37-60.

Braegelmann, Tom (2018): en Hartung, Markus, Bues, Micha-Manuel y Halbleib, Gernot (coords.), Legal Tech: die digitalisierung des rechtsmarkts (München, Beck) pp. 215- 230.

BRAND, Dirk (2020): "Algorithmic Decision-making and the Law", JeDEMeJournal of EDemocracy and Open Government, vol. 12, No 1: pp. 115-132.

BrauneIs, Robert, Goodman, Ellen (2018): "Algorithmic Transparency for the Smart City”, 20 Yale J.L. \& Tech, pp. 103-140.

Buat-Ménard, Eloi y Giambiasi, Paolo (2017): "La mémoire numérique des décisions judiciaires", Recueil Dalloz: p. 1483.

Buhmann, Alexander, Passmann, Johannes y Fieseler, Christian (2019): "Managing algorithmic accountability: Balancing reputational concerns, engagement strategie $s$, and the potential of rational discourse", Journal of Business Ethics. Disponible en: https:// doi.org/10.1007/s1055 1-019-04226-4 Fecha de consulta: 20 de febrero de 2021.

Buocz, Thomas (2018): "Artificial Intelligence in Court: Legitimacy Problems of AI Assistance in the Judiciary", Retskraft - Copenhagen Journal of Legal Studies, vol. 2, No 1, pp. 41-56.

Burrell, Jenna (2016): "How the machine 'thinks': Understanding opacity in machine learning algorithms", Big Data \& Society: pp. 1-25.

Busch, Christoph (2018): "Algorithmic Accountability", Abida Gutachten (Berlin, Bundes Ministerium für Bindulg und Forschung). 
Buttarelli, Giovanni (2018): "Choose Humanity: Putting Dignity back into Digital, Opening Speech of Debating Ethics", Public Session of the 40th Edition of the International Conference of Data Protection Commissioners, pp. 1-8.

CADiet, Loïc (2017): "L'open data des décisions de justice"., Rapport à Madame la garde des Sceaux, ministre de la Justice. Disponible en: http://www.jus- tice.gouv.fr/publication/ open data rapport.pdf Fecha de consulta: 20 de febrero de 2021.

Calliess, Gralf-Petter (2014): Der Richter im Zivil-prozess - Sind ZPO und GVG noch zeitgemäß? Gutachten A zum 70. Deutschen Juristentag (Hannover, München, Beck).

Campolo, Alex y Crawford, Kate (2020): "Enchanted determinism: Power without responsibility in artificial intelligence", Engaging Science, Technology, and Society, No 6: pp. 1-19.

CAPOnI, Remo (2015): "Il processo civile telematico tra scrittura e oralità", Rivista Trimestrale di Diritto e Procedura Civile, Anno LXIX, Fasc. 1, pp. 305-313.

Carneiro, Davide y otros (2014): "Online dispute resolution: an artificial intelligence perspective”, Artificial. Intelligence. Review, No 41: pp. 211-225 ss.

Chowdhury, Shammur y otros (2020): "Accessing the Ability of Personas to Alter Preconceptions of Audience Attributes", Proceedings of the HCI International (Copenhagen, Springer).

Citron, Danielle Keats (2008): “Technological Due Process”, 85 WASH. U. L. REV., pp. 1249-1269.

Cottin, Marianne (2019): "L’open data des décisions de justice: un bouleversement de l'espace jurisprudentiel ?", en Ferrand, Frédérique, KNeTsCh, Jonas y Zwickel, Martin (coords.), Die Digitalisierung des Zivilrechts und der Ziviljustiz in Deutschland und Frankreich (Erlangen, Fau U. Press) pp. 293-310.

Dalke, Dean (2013): “Can Computers Replace Lawyers, Mediators and Judges?”, The Advocate, No 9: pp. 703-720.

Dondero, Bruno (2017): “Justice prédictive: la fin de l'aléa judiciaire ?", Recueil Dalloz: pp. 532-530.

Doshi-Velez, Finale y Kim, Been (2017): “Towards A Rigorous Science of Interpretable Machine Learning”, ArXiv:1702.08608 [Cs, Stat].

Edwards, Lilian y Veale, Michael (2018): "Enslaving the algorithm: From a 'Right to an Explanation' to a 'Right to Better Decisions'?", IEEE Security and Privacy, vol. 16, No 3: pp. 46.54 .

ENDERS, Peter (2018): "Einsatz künstlicher Intelligenz bei juristischer Entscheidungsfindung”, Juristiche AusbildungA: pp. 721-733.

European Commission for the EfFiciency of Justice (2018): "European Ethical Charter on the Use of Artificial Intelligence in Judicial Systems and their environment", CEPEJ: pp. 16-36.

Felzmann, Heike y otros (2020): “Towards Transparency by Design for Artificial Intelligence", Science and Engineering Ethics, No 26: pp. 3333-3361.

Ferrand, Frédérique, Knetsch, Jonas y Zwickel, Martin (2019): "Introduction”, en FeRRAND, Frédérique, Knetsch, Jonas y Zwickel Martin (coords.), Die Digitalisierung des 
Zivilrechts und der Ziviljustiz in Deutschland und Frankreich (Erlangen, Fau U. Press) pp. 15-20.

Ferrario, Andrea, Loi, Michel y Viganò, Eleonora (2019): "In AI we trust incrementally. A multi-layer model of trust to analyze human-artificial intelligence interactions", Philosophy and. Technology, No 33: pp. 523-539.

Fersini, Elisabetta y otros (2014): "E-Mediation-Towards Smart Online Dispute Resolution”, KMISKnowledge Management Information System Conference Paper: pp. 228-236.

Fries, Martin (2016): "Man versus Machine: Using Legal Tech to Optimize the Rule of Law”. Disponible en https://ssrn.com/abstract=2842726. Fecha de consulta: 20 de febrero de 2021.

Fries, Martin (2018): “Legal Tech in der Justiz - Zeit für einen Robo Judge?”, Legal Tribune OnlineTO, vol. 9, № 3, https://www.lto.de/recht/justiz/j/legal-tech-digitalisierungziviljustiz-online-gerichtsstand-digitaler-richter/ Fecha de consulta 10 de febrero 2021.

Galdia, Marcus (2020): “The Comparative Element in Comparative Legal Linguistic", Comparative Legilinguistics, vol. 43: pp. 57-76.

GARAPON, Antoine (2017): "Les enjeux de la justice prédictive", JCP GJuris Classeur Periodique Generale: pp. 31-45.

Genn, Hazel (2012): "Why the Privatisation of Civil Justice is a Rule of Law Issue", 36th F.A. Mann Lecture, Lincoln's Inn, 19. 11: p. 16. Disponible en https://www.ucl.ac.uk/ laws/sites/laws/files/36th-f-a-mann-lecture-19.11. 12-professor-hazel-genn.pdf. Fecha de consulta: 20 de febrero de 2021.

Giacalone, Marco (2016): Dispute Resolution and New IT Realities. Tesis para optar al grado de Doctor de la Università di Napoli.

Gregor, Reinhard (2019): "Der Zivilprozess auf dem Weg in die digitale Sackgasse”, Neue Juristiche Wochenschrift NJW: pp. 3429-3435.

Gregor, Reinhard (2019): "Richterliche Prozessführung im digitalen Zeitalter", en Ferrand, Frédérique, Knetsch, Jonas y Zwickel, Martin (coords.), Die Digitalisierung des Zivilrechts und der Ziviljustiz in Deutschland und Frankreich (Erlangen, Fau U. Press) pp. 267-280.

GÜNTHER, Jan Philipp (2016): Roboter und rechtliche Verantwortung (Münchenm Herbert Utz Verlag).

Huber, Steffan y Giesecke, Tim (2020): "Einführung” en Ebers, Martin y otros (coord.), Künstliche Intelligenz und Robotik (München, Beck) pp. 2-20.

IsHII, Kaori (2019): "Comparative legal study on privacy and personal data protection for robots equipped with artificial intelligence: looking at functional and technological aspects", AI \& Society, № 34: pp. 509-533.

Jolowicz, John (2000): On Civil Procedure (Cambridge, Cambridge University Press).

Kaminski, Margot (2019): "The Right to Explanation, Explained", Berkeley Technology Law Journal, vol. 34: pp. 189-217.

KAUSHIK y otros (2020): "Explaining the Efficacy of Counterfactually-Augmented Data", ArXiv:2010.02114 [Cs, Stat]. 
Krans, Burt, Nylund, Anna (2021): "Civil Courts Coping with Covid-19 - Exceptional Times, Normal Times, New Times?”, en Krans, Burt, Nylund, Anna (Coords.), Civil Courts Coping with Covid-19 (The Hague, Elven International Pubblishing) pp. 1-5.

Krause, Paul, Fox, John y Judson, Philip (1993). "An argumentation-based approach to risk assessment”, IMA Journal. of Management Mathematics, vol. 5, № 1 5.1: pp. 249-263.

LARSON y otros (2016): "How we analyzed the compass recidivism algorithm", ProPublica. Disponible en: https://www.propublica.org/article/how-we-analyzed-the-compas-recidivism-algorithm. Fecha de consulta: 20 de febrero de 2021.

LEE y otros (2019): "Procedural Justice in Algorithmic Fairness: Leveraging Transparency and Outcome Control for Fair Algorithmic Mediation", Proceedings. of the ACM on the Human-Computert. Interaction, vol. 3, No. CSCW, Article 182: pp. 1-24.

Lodder, Aarno y Thiessen, Ernest (2013). "The Role of Artificial Intelligence in Online Dispute Resolution”. Disponible en: http://www.mediate.com/Integrating/docs/lodder_ thiessen.pdf. Fecha de consulta: 20 de febrero de 2021.

Looks, Moshe y LouI, Ronald (2005). "Game Mechanisms \& Procedural Fairness”, Conference Paper JURIX: pp. 1-10.

Malgieri, Gianclaudio (2019): "Automated Decision-Making in the EU Member States: The Right to Explanation and Other "Suitable Safeguards" in the National Legislations”, Computer Law \& Security Review, vol. 35, No 5, Elsevier 105327, pp. 1-26.

McFadden, Trevor (2019): "Can AI Be a Fair Judge in Court? Estonia Thinks so", Wired. Disponible en: https:/www.wired.com/story/can-ai-be-fair-judge-court-estonia-thinksso/. Fecha de consulta: 20 de febrero de 2021.

Millar, Jason y Kerr, Ian (2016): "Delegation, relinquishment, and responsibility: The prospect of expert robots", en Calo, Ryan, Froomkin, Michael y Kerr, Ian (coords.), Robot Law (London, Edward Elgar Publishing) pp. 104-118.

Mitrou, Lilia (2019): "Artificial Intelligence and Cognitive Services: Is the General Data Protection Regulation (GDPR) Artificial Intelligence-Proof'?”. Disponible en: https:// ssrn.com/abstract=3386914. Fecha de consulta: 20 de febrero de 2021.

Pégny, Maël, Thelisson, Eva y Ibnouhsein, Issam (2019): “The Right to an Explanation”, Delphi, No 4: pp. 161-178.

Pérez Ragone, Álvaro (2020): "Justicia Artificial: desafíos y oportunidades”, REVISTA DE PROCESSO. San Pablo, Thomson Reuter-Revista dos Tribunais. vol. 301: pp. 401-419.

Perry, Melissa (2017): “iDecide: Administrative Decision-Making in the Digital World”, 91 Australian Law Journal: pp. 29-50.

PIroteK, Christian (2018): "Vorbild Dänemark: Digitalisierung des Zivilprozesses". Disponible en: https://www.cmshs-bloggt.de/dispute-resolution/litigation/vorbild-daenemark-digitalisierung-deszivilprozesses/. Fecha de consulta: 20 de febrero de 2021.

PRÜTTING, Hanns (2018): "Rückgang der Klageeingangszahlen bei den staatlichen Gerichten", DRiZDeutsche Richterzeitung: pp. 62-75.

Richards, Neil y Smart, William (2016): "How Should the Law Think About Robots?", en Calo, Ryan, Froomkin, Michael, Kerr, Ian (coords.), Robot Law (London, Edward Elgar Publishing) pp. 5-7. 
RÜHL, Gisela (2020): “Digitale Justiz, oder: Zivilverfahren für das 21. Jahrhundert”, JuristenZeitung, No 75: pp. 809-817.

RÜHL, Gisela (2020): "KI in der gerichtlichen Streitbeilegung”, en Braegelmann, Tom y Kaulartz, Markus (coords.), Rechtshandbuch Künstliche Intelligenz und Machine Learning (München, Beck) pp. 617-627ss.

Rule, Colin (2017): "Designing a Global Online Dispute Resolution System: Lessons Learned from eBay”, St. Thomas Law. Journal, No 13: pp. 354 y 366.

Salminen, Joni y otros (2020): "Persona Transparency: Analyzing the Impact of Explanations on Perceptions of Data-Driven Personas", International Journal of Human-Computer Interaction, vol. 36, No 8: pp. 788-800.

SAlter, Shannon (2017): "ODR and Justice System Integration: B.C.'s Civil Resolution Tribunal". Windsor Yearbook of Access to Justice, No 34: pp. 112-120.

SAmeK, Wojciech y Müller, Klaus-Robert (2019). “Towards Explainable Artificial Intelligence”, en SAMEK, Wojciech y otros (coords.), Explainable AI: Interpreting, Explaining and Visualizing Deep Learning (Suiza, Springer International Publishing) pp. 5-22.

Sauvé, Jean Marc (2018): Ordre des avocats au Conseil d'État et à la Cour de cassation, La justice prédictive. Actes du colloque du 12 février (Paris, Dalloz).

Schwab, Klaus (2016): "The Fourth Industrial Revolution: what it means and how to respond”. Disponible en: www.weforum.org/agenda/2016/01/the-fourth-industrial-revolution-what-it-means-and-how-to-re-spond/. Fecha de consulta: 20 de febrero de 2021.

Selbst, Andrew y Powles, Julia (2017): "Meaningful Information and the Right to Explanation”, International Data Privacy Law, vol. 7, No 4: pp. 233-242.

Specht, Florian (2019): "Chancen und Risiken einer digitalen Justiz für den Zivilprozess", Multimedia \& RechtMR: pp. 153-162.

STADLER, Astrid (2020): "Grenzen der Inkassozession nach dem Rechtsdienstleistungsgesetz”. Besprechungsaufsatz zu BGH, Urt. v. 27.11.2019, VIII ZR 285/18, JZ 7/2020: pp. 321-331.

Statistisches Bundesamt (2019): "Fachserie 10 Reihe 2.1 Rechtspflege", Zivilgerichte: pp. 12-20.

STEFFEn, Till (2018): "Recht muss auch im Kleinen wirken", DRiZDeutsche Richterzeitung: pp. 334- 350.

Sourdin, Tania (2015): “The Role of the Courts in the New Justice System”, 7 Yearbook on Arbitration and Mediation, pp. 95-116.

Sourdin, Tania (2018): "Judge v Robot? Artificial Intelligence and Judicial Decision-Making”, UNSW Law Journal, Vol. 4, No 4: pp. 1114-1133.

Susskind, Richard (2017): Tomorrow's Lawyers. An Introduction to Your Future (Oxford, Oxford. U. Press).

Susskind, Richard (2019): Online Courts and the Future of Justice (Oxford, Oxford U. Press).

Teuber, Gunther (2018): “Mensch-Maschinen-Verbünden und Multi-Agenten-Systemen”, Archiv für Civilistiche Praxis: pp. 155-198. 
Thevenot, François (2019): "L'open data et justice prédictive", en Ferrand, Frédérique, Knetsch, Jonas y Zwickel, Martin (Coords.), Die Digitalisierung des Zivilrechts und der Ziviljustiz in Deutschland und Frankreich (Erlangen, Fau U. Press).

Tosoni, Luca (2021): “The Right to Object to Automated Individual Decisions: Resolving the Ambiguity of Article 22(1) of the General Data Protection Regulation”, International Data Privacy Law, No 24.

Trabajo grupal Legal Tech (2019): “Herausforderungen für die Justiz". Disponible en: https://www.justiz.nrw.de/JM/jumiko/beschluesse/2019/Fruehjahrskonferenz_2019/ index.php. Fecha de consulta: 20 de febrero de 2021.

Treleaven, Philip, Barnett, Jeremy y Soares Koshiyama, Adriano (2019): "Algorithms: Law and Regulation", Computer. Disponible en: www.computer.org/csdl/magazine/ co/2019/02. Fecha de consulta: 20 de febrero de 2021.

UlenAers Jasper (2020): “The Impact of Artificial Intelligence on the Right to a Fair Trial: Towards a Robot Judge?”, Asian J. Law Econ, vol. 11, No 2: pp. 2-40.

VoGL, Roland (2018): "Changes in the US Legal Market Driven by Big Data/ Predictive Analytics and Legal Platforsm", en Hartung, Markus, Bues, Micha-Manuel y Halbleib, Gernot (coords.), Legal Tech: die digitalisierung des rechtsmarkts (München, Beck) pp. 53-56.

Wachter, Sandra, Mittelstadt, Brent y Floridi, Luciano (2017): “Transparent, explainable, and accountable AI for robotics", 2/6 Science Robotics.

Wachter, Sandra, Mittelstadt, Brent y Floridi, Luciano (2017): "Why a Right to Explanation of Automated Decision-Making Does Not Exist in the General Data Protection Regulation”, International Data Privacy Law, vol. 7, No 2: pp. 76-99.

Wagner, Jens, (2018): Legal Tech und Legal Robots (Wiesbaden, Springer).

Waldman, Ari Ezra, (2019): "Power, Process, and Automated Decision-Making", Fordham Law Review, vol. 88, No 2: pp. 613-632.

Warren, Agin (2018): "Using Machine Learning to Predict Success or Failure in Chapter 13 Bankruptcy Cases”, Annual Survey of Bankrupcy. Law, No 13 (2018 WL 4293106).

Young, Chris (2019): “China Has Unveiled an AI Judge that Will 'Help' With Court Proceedings”. Disponible en: https://interestingengineering.com/china-has-unveiled-an-aijudge- that-will-help-with-court-proceedings. Fecha de consulta: 20 de febrero de 2021.

Zarsky, Tal (2013): “Transparent Predictions”, University of Illinois Law Review, vol. 68, No 4: pp. 1503-1570.

Zerilli, John (2018): "Transparency in Algorithmic and Human Decision- Making: Is There a Double Standard?", Philosophy \& Technology. Disponible en: https://doi. org/10.1007/s13347-018-0330-6. Fecha de consulta: 20 de febrero de 2021.

Zwickel, Martin (2018): "Die digitale Strukturierung und inhaltliche Erschließung zivilprozessualer Schriftsätze im Spannungsfeld zwischen Parteiherrschaft und Richtermacht”, en Buschmann, Almuth y otros (coords.), Digitalisierung der gerichtlichen Verfahren und das Prozessrecht - 3. Tagung junger Prozessrechts-wissenschaftler und -wissenschaftlerinnen am 29.130.09.2017, p. 179-190. 
Zuckerman, Adrian (2020): "Artificial Intelligence in the Administration of Justice", en Higgins, Andrew (Coord.), The Civil Procedure Rules at 20, (Oxford, Oxford University Press), pp. 291-304.

\section{NORMAS CITADAS}

Gesetz Zu Verwendung elektronischer Kommunikationsformen in der Justiz (2005): $B G B l$, vol. 22: p. 837.

Gesetz zur Anpassung der Formvorschriften des Privatrechts und anderer VorsCHRIFTEN AN DEN MODERNEN RECHTSGESCHÄFTSVERKEHR (2001): BGBl, vol. 13: p. 1542.

Gesetz zur Einführung der eleKtronischen AKte in Der Justiz und ZUR Weiteren Förderung des elektronischen Rechtsverkehrs (2017): BGBl, vol. 5: p. 2208.

Gesetz zur Förderung des elektronischen Rechtsverkehrs mit den Gerichten (2013): $B G B l$, vol. 10: p. 3786 .

Gesetz Zur Reform des Verfahrens bei Zustellungen im gerichtlichen Verfahren (2001): $B G B l$, vol. 25: p. 1206.

Gesetz Zur Reform des Zivilprozesses (2001): $B G B l$, vol. 27: p. 1887.

Loi No 2016-1321 de 2016, RéPUbliQue NUMÉRIQUe.

Ley No 20.886 de 2015, Modifica el Código de Procedimiento Civil, Para establecer la TRAMITACiÓn DigITAL DE los PROCEDIMIENTOS JUdiCIALES.

Directiva No 2013/11/EU del Parlamento Europeo y del Consejo, de 2013, relativa a LA RESOlUCIÓN ALTERNATIVA DE LITIGIOS EN MATERIA DE CONSUMO Y POR LA QUE SE MODIFICa el Reglamento No 2006/2004 y la Directiva No 2009/22/CE.

ZIVILPROZESSORDNUNG ALEMAN.

\section{JURISPRUDENCIA CITADA}

Alemania

Supremo Tribunal Federal alemán en BGH NJW-RR (2009) pp. 357, 358.

\section{SITIOS WEB INSTITUCIONALES VISITADOS}

https://www.online-schlichter.de Fecha de consulta 10 de febrero 2021. https://www.ombudsmann.at. Fecha de consulta 10 de febrero 2021. https://verbraucher-schlichter.de/. Fecha de consulta 10 de febrero 2021. https://smartsettle.com/products/smartsettle-one/. Fecha de consulta 10 de febrero 2021. https://www.ris.bka.gv.at/. Fecha de consulta 10 de febrero 2021. 
